\title{
Le tourisme coopératif au Québec : une forme de tourisme vecteur de développement pour les territoires périphériques?
}

Cooperative tourism in Quebec: a new form of tourism, a vector of development for peripheral territories?

El turismo cooperativo en Quebec : ¿una nueva forma de turismo, vector de desarrollo para los territorios periféricos?

Sylvain Salaméro, Gilles Caire et Christiane Gagnon

\section{OpenEdition}

\section{Journals}

Édition électronique

URL : https://journals.openedition.org/ideas/3390

DOI : $10.4000 /$ ideas.3390

ISSN : $1950-570$

Éditeur

Institut des Amériques

Référence électronique

Sylvain Salaméro, Gilles Caire et Christiane Gagnon, « Le tourisme coopératif au Québec : une forme de tourisme vecteur de développement pour les territoires périphériques? », IdeAs [En ligne], 12 | 2018, mis en ligne le 21 novembre 2018, consulté le 18 octobre 2022. URL : http://journals.openedition.org/ ideas/3390 ; DOI : https://doi.org/10.4000/ideas.3390

Ce document a été généré automatiquement le 18 octobre 2022

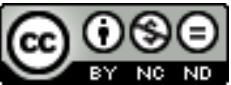

Creative Commons - Attribution - Pas d'Utilisation Commerciale - Pas de Modification 4.0 International - CC BY-NC-ND 4.0

https://creativecommons.org/licenses/by-nc-nd/4.0/ 


\section{Le tourisme coopératif au Québec : une forme de tourisme vecteur de développement pour les territoires périphériques?}

Cooperative tourism in Quebec: a new form of tourism a vector of development for peripheral territories?

El turismo cooperativo en Quebec: ¿una nueva forma de turismo, vector de desarrollo para los territorios periféricos?

Sylvain Salaméro, Gilles Caire et Christiane Gagnon

1 Depuis une quarantaine d'années, différentes formes de tourisme ont émergé : tourisme durable, écotourisme, agritourisme, tourisme solidaire ou tourisme communautaire (Lequin M., 2001 ; Gagnon C. et Gagnon S., 2007 ; Laurent A., 2009 ; Breton J.-M., 2010), insistant sur la place des populations locales tant dans les processus de mise en tourisme que dans leur appropriation des bénéfices (Gagnon C. et Fortin M.J., 1999 ; Lequin M., 2001 ; Delisle M.-A. et Jolin L., 2007 ; Schéou B., 2009). De même, la participation de ces populations, dans la prise de décision et l'aménagement, est souvent présentée par les institutions internationales et nationales comme une solution pour initier, en zone périphérique, un tourisme vecteur de développement durable des territoires. Ces formes de tourisme visent à favoriser des retombées économiques pour les territoires (Gagnon C., 1999 ; Caire G.,2006 ; Delisle M.-A. et Jolin L., 2007). Plus discrètes en Europe, les formes de tourisme communautaire ${ }^{1}$ se développent davantage en Amérique du Nord, notamment avec les initiatives liées au tourisme autochtone. Selon Katia Iankova (2005), près de $70 \%$ des communautés autochtones québécoises planifient des activités touristiques. Parmi elles, les communautés inuits ont privilégié la formule coopérative.

2 Toutefois, les coopératives touristiques dépassent très largement le champ du tourisme autochtone : plus de 200 coopératives touristiques œuvrent dans le secteur du tourisme 
et du loisir (récréotourisme) sur l'ensemble du territoire québécois. Curieusement, peu de travaux ont documenté cette forme de tourisme qui est en pleine croissance. Quel est le statut de ces coopératives touristiques? Où se localisent-elles? Quel poids représentent-elles dans les régions du Québec ? Quelles sont leurs spécificités ? En quoi sont-elles conformes ou non aux principes de l'économie sociale? Voilà autant de questions qui sous-tendent des objectifs de recherche et une problématique liée au développement territorial et au poids du tourisme dans celui-ci. L'hypothèse centrale est que les coopératives touristiques constituent un modèle pertinent de tourisme géré par les acteurs locaux et, du coup, une avenue de développement territorial. L'objectif principal de recherche est d'améliorer les connaissances, sur cette forme de tourisme peu documentée, selon une démarche méthodologique, quantitative et qualitative, de classement et d'analyse statistique et cartographique à l'échelle des dix-sept régions du Québec.

3 Cet article, après avoir rappelé le contexte géo-historique et sociétal singulier d'émergence du tourisme coopératif (1) et la méthodologie mobilisée dans cette recherche (2), vise à dresser un état des lieux - sous les angles statistique (3), territorial (4) et des caractéristiques le rattachant au champ de l'économie sociale (5) - et à discuter des résultats (6).

\section{La culture coopérative au Québec : un contexte déterminant}

4 Le succès des coopératives touristiques s'inscrit dans une longue tradition d'économie sociale au Québec, ce qui la distingue du reste du Canada ${ }^{2}$ (Vaillancourt Y., 2008). En effet, les premières associations ouvrières et sociétés de secours mutuel ont émergé dès la fin du XVIII ${ }^{e}$ siècle $^{3}$ et leur développement a été continu à partir de 1850 . Toutefois, elles péricliteront à la fin du XIX ${ }^{e}$ siècle par manque de reconnaissance et de soutien de l'État québécois, suite aux pressions de l'Église (Lévesque B., 2007a). La tradition sociale-chrétienne, qui prônait «à des degrés divers le corporatisme social, la collaboration entre patron et travailleurs (patronage) et la doctrine sociale de l'Église " prit le relais (D'Amours M., 2006: 60). Elle influença l'émergence des coopératives agricoles ainsi que les premières coopératives financières. Alphonse Desjardins, le fondateur de la Fédération des caisses populaires, qui est devenue l'un des plus grands groupes coopératifs mondiaux avec près de 248 milliards de dollars canadiens d'actifs, a puisé son inspiration dans cette doctrine sociale catholique (Ibid).

5 Par ailleurs, la crise économique des années 1930 eut pour conséquence un important développement du mouvement coopératif encouragé par les élites locales catholiques, notamment dans les secteurs de la consommation, de la pêche et de la forêt. Les coopératives ont émergé pour répondre à des besoins non résolus par l'État ou le marché. Parmi cette génération de coopératives, certaines intégreront des activités touristiques à l'instar de la Coopérative de Transport Maritime et Aérien (CTMA). Celleci est créée en 1944. Les habitants des îles-de-la-Madeleine (territoire le plus périphérique du Québec dans le Golfe du Saint-Laurent) font alors face à un problème récurrent d'approvisionnement par voie maritime. À l'initiative d'Ovide Hubert, inspecteur d'écoles, des commerçants, des coopératives de pêche et des magasins coopératifs se sont regroupés pour investir dans cette coopérative. C'est aujourd'hui la 
seule coopérative de transport maritime en Amérique du Nord. Elle est devenue le transporteur exclusif pour les îles de la Madeleine ${ }^{4}$.

6 À partir de 1960, avec la Révolution tranquille, l'État québécois se constitue et se distancie de l'Église. Animé par un courant d'identité nationale, il se modernise et intervient dans les sphères sociale et économique. L'affirmation de l'État, sa déconfessionnalisation et la nationalisation de certains secteurs névralgiques, dont l'hydroélectricité, éclipseront momentannément le mouvement coopératif.

7 À l'inverse, les années 1970 «représentent l'âge d'or des groupes populaires de services $»^{5}$ (D'Amours M., $2006:$ 73) qui luttent contre l'exclusion en s'appuyant sur les populations locales et le développement de services communautaires autogérés (Favreau L., 2010). Ce « développement économique communautaire» est inspiré de l'approche "community-based economic development», popularisée au Canada et aux États-Unis. Ainsi des objectifs économiques et sociaux sont intégrés dans la constitution de coopératives de travail, de groupes de travailleurs actionnaires, de groupes communautaires et de fonds de financement ; ils expérimentent des solutions face à la crise des années 1980 et le désengagement de l'État providence (Lévesque B. et Mendell M., 2005).

8 À partir des années 1980, les syndicats québécois opérent un virage stratégique : ils participent à la reprise d'entreprises par les travailleurs. Cela conduira à la création de coopératives de travailleurs comme Premier défi, Laval, ${ }^{6}$ et à des initiatives de développement économique.

9 En outre, le mouvement coopératif se renouvelle avec l'arrivée d'une nouvelle génération de coopératives plus ancrées localement (D’Amours M., 2006). Leurs activités sont axées sur les services de proximité, tels que des coopératives de consommation, funéraires, de logements locatifs, des garderies. Il existe aussi des coopératives amérindiennes et inuites, l'une des plus emblématiques étant celle de la réserve des Innus d'Essipit avec une offre touristique diversifiée. Amputés d'une partie de leur territoire en raison du développement de l'industrie forestière, les Innus ont en effet dès le début du $\mathrm{XX}^{\mathrm{e}}$ siècle favorisé la commercialisation de produits d'artisanat auprès des villégiateurs fréquentant Tadoussac. En 1978, la communauté s'est lancée dans le développement d'une offre récréotouristique, en particulier l'observation des baleines et les pourvoiries ${ }^{7}$, avec la création des Entreprises Essipit privilégiant en même temps un système coopératif communautaire par le réinvestissement des profits dans la mise en place d'infrastructures (éoliennes, conserverie) et de services collectifs ${ }^{8}$.

10 Dans les années 1990, le militantisme économique de la société civile et des mouvements sociaux ${ }^{9}$ a impulsé un nouveau mode d'institutionnalisation qui repose sur la participation et la consultation des acteurs de l'économie sociale par l'état québécois (Lévesque B. et Mendell M., 1999). En outre, la Loi sur les coopératives (1997) introduit le statut de coopérative de solidarité. Ce statut, inspiré des coopératives sociales italiennes, est particulièrement adapté aux services de proximité et repose sur une gouvernance partenariale de ces organisations puisque sont réunis, à la même table, les travailleurs, les usagers et des membres de la population locale.

11 Ce contexte et tous ces changements institutionnels ont influencé profondément le tourisme coopératif des vingt dernières années et ainsi favorisé son essor, à l'instar de la Coopérative de solidarité en tourisme équitable (Coste) ${ }^{10}$. A partir de 2006, les acteurs locaux de la Côte-Nord en quête de diversification économique, ont commencé par se 
concerter pour assurer la mise en tourisme d'une région éloignée des centres urbains. Cette concertation a abouti en 2009 à la création d'une agence de voyages. Soutenue par diverses institutions gouvernementales (ministères du Tourisme, du Développement économique de l'Innovation et de l'Exportation) et régionales (Centres locaux de développement de la Basse-Côte-Nord et de la Minganie, Conférence régionale des élus), la coopérative coste rassemble une soixantaine de membres, tels que des municipalités locales, des compagnies d'aviation, des établissements hôteliers, la Sépaq (Société des établissements de plein air du Québec).

\section{Une méthodologie particulière pour appréhender un objet peu documenté à l'échelle des régions du Québec}

12 Ce constat d'un renouveau coopératif, décliné ici pour le tourisme, nous a interrogés. Ces initiatives de mise en tourisme repérées au Québec émanent d'acteurs locaux et sont, pour une majorité d'entre elles, localisées dans des territoires non métropolitains. Elles adoptent des statuts particuliers, la plupart avec une gouvernance multipartite, dans le champ de l'économie sociale. Le peu d'études scientifiques et de littérature professionnelle sur ce phénomène social a forcé l'adoption d'une posture exploratoire et descriptive, divisée en trois phases. La première a consisté à contextualiser le tourisme coopératif en dégageant les dynamiques territoriales, coopératives et touristiques générales dans les dix-sept régions québécoises de 1997 à $2013^{11}$. La seconde a consisté à dresser un état des lieux du tourisme coopératif au Québec, notamment selon les dynamiques de localisation et leurs spécificités en particulier par rapport à l'ensemble des coopératives non financières et au tourisme en général sur la même période. En outre, une analyse documentaire qualitative a été réalisée pour recenser les activités de ces coopératives. La troisième phase de recherches fut consacrée à répondre aux questions soulevées à l'issue des deux premières phases en conduisant une analyse à un niveau scalaire plus fin. Les deux principaux outils alors mobilisés sont l'analyse cartographique et la technique du quotient de localisation ${ }^{12}$ (Béguin et Pumain, 2014). Ces outils se sont avérés adaptés à une démarche exploratoire de classement, de dénombrement et de cartographie; ils ont permis de répondre aux questions de recherche, identifiées dans l'introduction.

\section{État des lieux statistique du tourisme coopératif québécois}

De l'état des lieux réalisé dans le cadre de la présente recherche, quatre constats principaux se dégagent. En terme de chiffres, un premier constat s'impose : en 1997, l'année de l'adoption de la Loi sur les coopératives, 80 coopératives touristiques étaient dénombrées au Québec. Il en existait 209 en 2014. Entre 2001 et 2013, leur nombre a augmenté de près de $80 \%$. En comparaison, les coopératives non financières n'ont progressé que de $4 \%$. Cette augmentation du nombre des coopératives touristiques est également remarquable au regard d'un contexte touristique morose sur cette période au Québec ${ }^{13}$. 
Le second constat est le succès du modèle de coopérative de solidarité à gouvernance multipartenariale ${ }^{14}$, comparé à une relative stabilité, voire même un déclin des statuts coopératifs plus anciens. Au Québec, le développement de cette nouvelle forme de coopérative explose. En effet, alors que celles-ci étaient marginales en 1996, elles représentent en 2013 près de $18 \%$ des coopératives non financières, $20 \%$ de leurs membres et $10 \%$ des emplois. Dans le secteur touristique, leur succès est sans équivoque : quasi inexistantes en 1997, plus de la moitié des coopératives touristiques ont adopté le statut de coopérative de solidarité en 2013 (cf. graphique 1.).

Graphique 1 - Évolution du nombre de coopératives touristiques par statut 1996-2013

Source : MÉSI, 2016

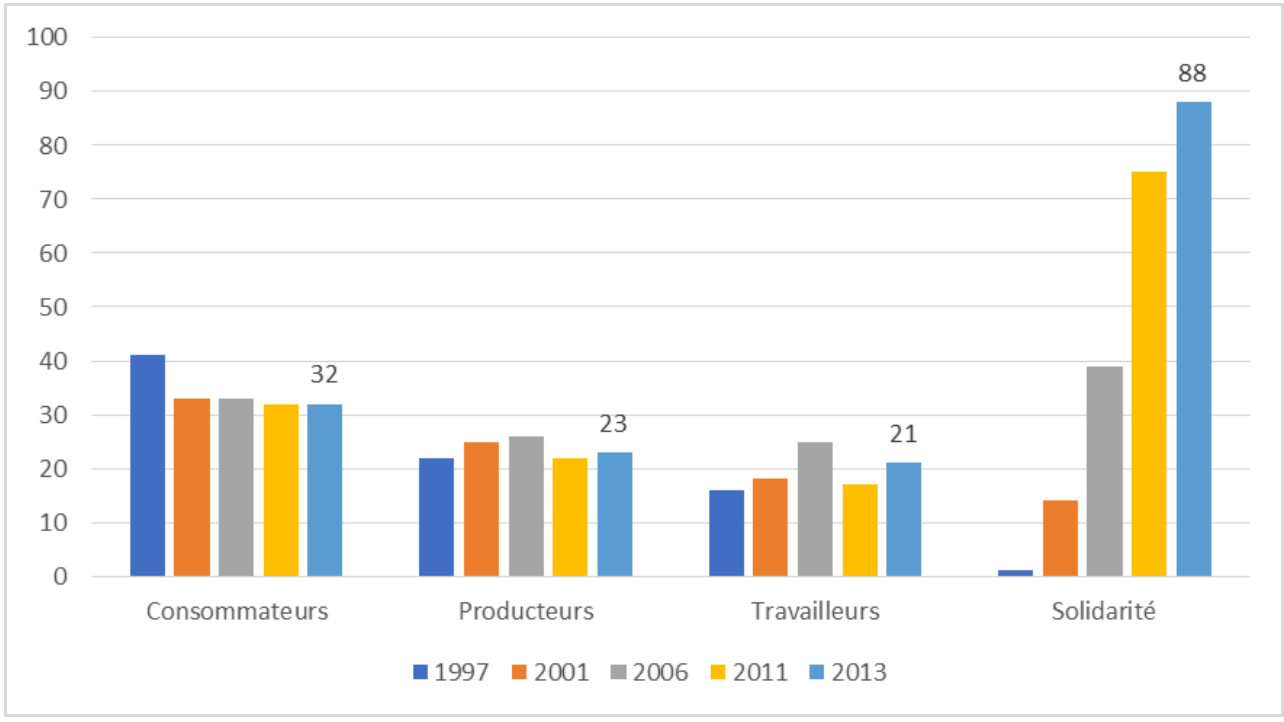

Ces coopératives de solidarité regroupaient alors $70 \%$ des membres de l'ensemble des coopératives touristiques et généraient $32 \%$ des emplois. Le succès de ce modèle coopératif compense la perte de membres des modèles coopératifs plus classiques observée de 1997 à 2013. Les coopératives de travailleurs représentaient la moitié des emplois du tourisme coopératif en 1997. À la fin de la période d'observation, elles ne génèrent que $27 \%$ des emplois.

Un troisième constat est identifié. Les coopératives touristiques, mis à part les coopératives de transport, correspondent généralement à des petites entreprises avec un chiffre d'affaires plus faible et avec moins d'actifs que dans l'ensemble du mouvement coopératif.

Enfin, un quatrième constat est que les coopératives de solidarité semblent générer des emplois moins rémunérés que les organisations plus traditionnelles. Cela étant, les salaires chargés moyens par emploi des coopératives touristiques de solidarité ont pratiquement doublé entre 2001 et 2011. Ces dernières sont relativement récentes, en moyenne 6 ans d'ancienneté (chiffre 2013). Il est probable que leur consolidation entraîne cette amélioration des rémunérations. 


\section{Un tourisme coopératif plus présent dans les régions périphériques québécoises}

l'étude révèle qu'elles se localisent principalement dans les régions éloignées des deux grands pôles urbains de Montréal et Québec. Au regard des données statistiques concernant les différentes dynamiques régionales, quatre résultats de recherche sont dégagés.

premier lieu, les coopératives touristiques se développent davantage dans les régions dynamiques sur le plan coopératif, telles que Bas-Saint-Laurent, Centre-duQuébec, Gaspésie-Îles-de-la-Madeleine et Saguenay-Lac-Saint-Jean. Ces régions sont souvent des régions en difficulté, c'est-à-dire où les indicateurs classiques de développement sont en-dessous de la moyenne québécoise (cf. Carte 1).

En second lieu, les coopératives touristiques se situent principalement dans les régions touristiques, à l'exception de celles où le tourisme marchand est très présent, telle celle des Laurentides, région à proximité du grand bassin montréalais. Ainsi les deux régions les plus dynamiques en matière de tourisme coopératif sont la Gaspésie-îles-de-laMadeleine et le Saguenay-Lac-Saint-Jean (cf. Carte 1).

Carte 1 - L'économie touristique et l'économie coopérative mobilisées dans les régions administratives québécoises en difficulté démoéconomique.

Cartographie : Salaméro, 2016.

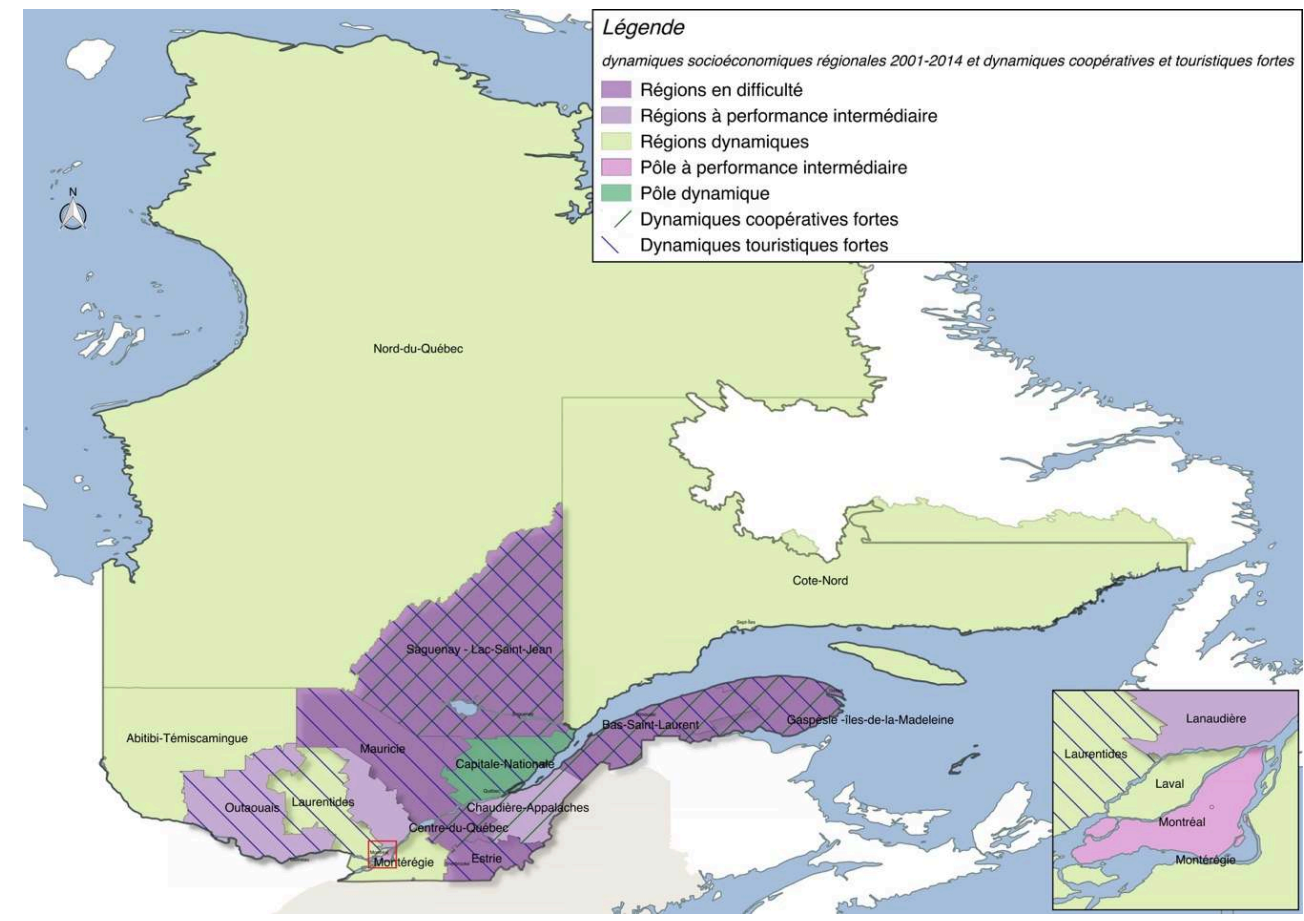

Un troisième résultat démontre que les coopératives touristiques se localisent particulièrement dans les régions périphériques, et notamment dans celles qui connaissent des difficultés démographiques et économiques ${ }^{15}$. De plus, les retombées économiques sont plus concentrées dans ces territoires en particulier au chapitre de la création d'emplois ${ }^{16}$. Il existe donc un effet de mobilisation coopératif et touristique 
dans les territoires en difficulté. Enfin un dernier résultat, et non le moindre compte tenu du discours politique sur les retombées économiques mirobolantes du tourisme, révèle que malgré cette concentration, ces retombées demeurent faibles à l'échelle régionale. L'effet de mobilisation ne permet pas d'inverser la tendance en matière de dynamiques régionales démoéconomiques en déclin, pendant la période d'observation. Bien sûr, une analyse à une échelle trop large pourrait masquer d'éventuels effets plus localisés, ce qui conduit à proposer une méthode d'analyse à un niveau scalaire plus fin. Les hauts-lieux touristiques peuvent être mis en évidence en géocodant la base des données sur les lieux touristiques de Tourisme Québec ${ }^{17}$. En postulant que l'offre correspond de manière significative à la demande, la cartographie de la densité de cette offre permet, dans une certaine mesure, d'appréhender l'attractivité touristique de ces sites. Grâce à la technique de la carte de chaleur (cf. carte 2), les hauts-lieux touristiques ont été cartographiés en faisant abstraction du maillage administratif par région, lequel ne correspond pas forcément au déploiement géographique du tourisme.

Carte 2 - Principaux hauts lieux touristiques au Québec en 2015 (Focus)

Source : Tourisme Québec 2015 - Cartographie : Salaméro, 2015

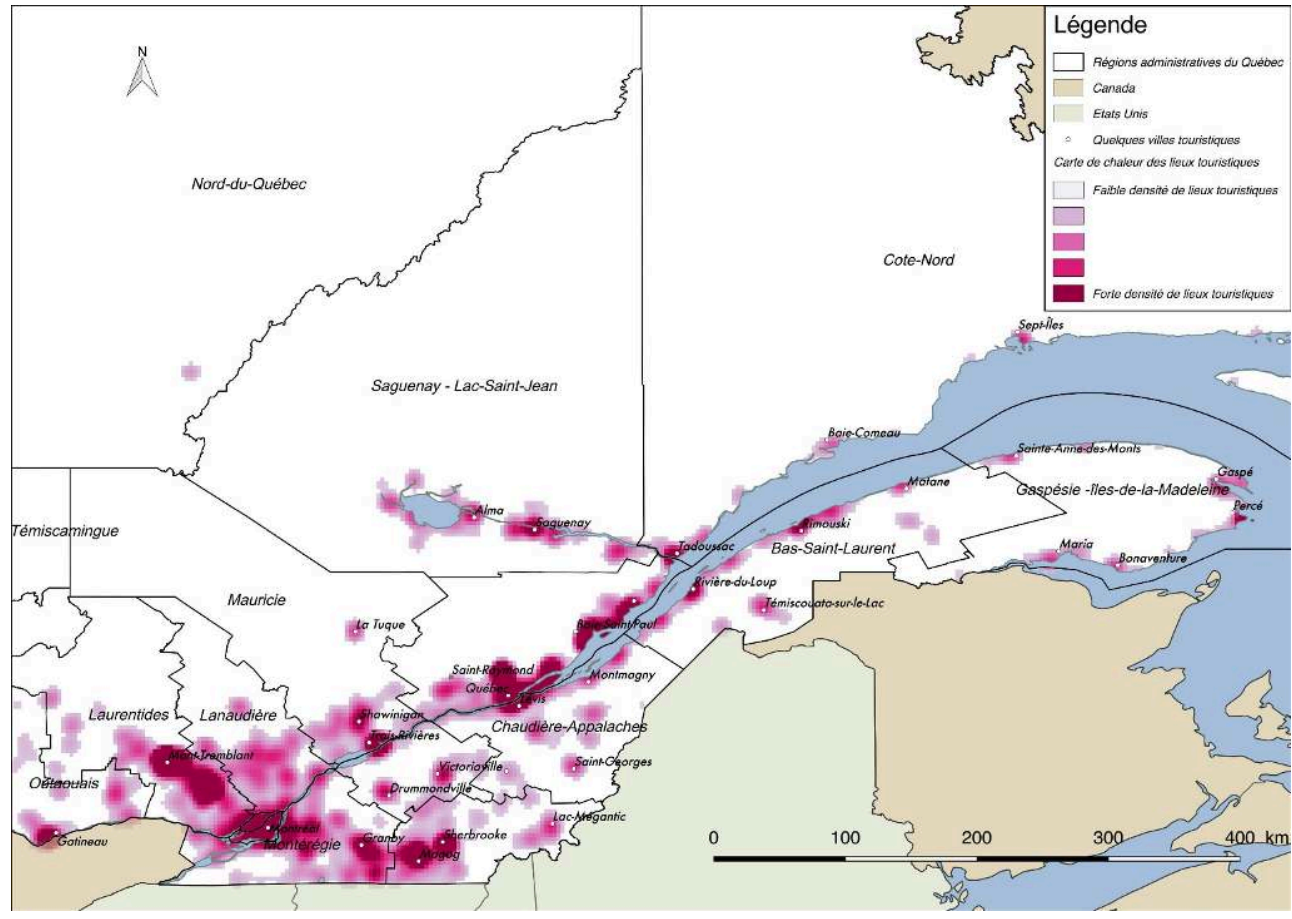

De même, les hauts-lieux coopératifs ont été cartographiés. Par la suite, les dynamiques démographiques ont été croisées avec les données sur les revenus disponibles (2001-2014) pour identifier les territoires qui se développent, selon cet indicateur, et les territoires en difficulté en matière de peuplement et de captation de revenus. Cela a permis de confirmer et de valider les quatre résultats précédents obtenus à l'échelle régionale: les coopératives touristiques se sont localisées majoritairement dans les hauts-lieux touristiques, dans $56 \%$ des cas, et les hauts-lieux coopératifs dans $57 \%$ des cas, et pour $47 \%$ d'entre elles, dans des zones à la fois touristiques et coopératives. Il existe donc un effet de proximité touristique et coopératif. De plus, elles sont plus concentrées dans les municipalités qui connaissent des difficultés démoéconomiques ${ }^{18}$. De même, le manque de retombées du tourisme coopératif en matière de 
développement démographique et économique, observé à l'échelle régionale, se retrouve aussi à l'échelle microrégionale.

Carte 3- Localisation des coopératives touristiques par rapport aux hauts lieux touristiques et coopératifs

Source : MÉSI, 2016 et Tourisme Québec, 2015 - Cartographie : Salaméro, 2016

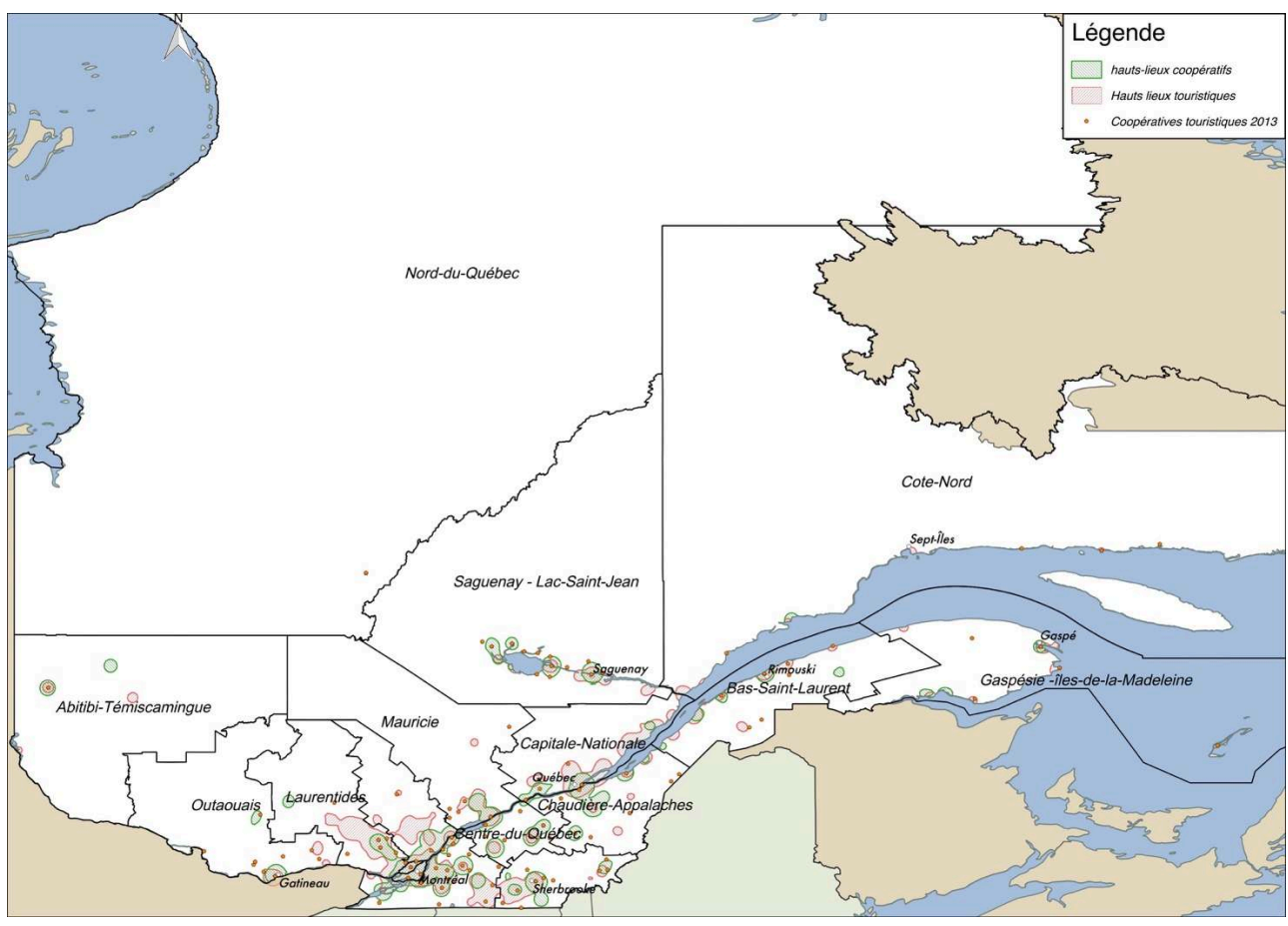

$\mathrm{Au}$ Québec, le tourisme coopératif se localise donc principalement dans les territoires en difficulté démoéconomique, mais qui disposent de dynamiques coopératives et touristiques fortes.

\section{La différenciation et les quatre spécificités du tourisme coopératif québécois}

Le tourisme coopératif se différencie des autres formes formes de tourisme commutauraire selon deux dimensions. La première concerne la réponse à des besoins sociospatiaux en matière de création d'emplois et de réinsertion sociale. Le nombre d'emplois générés est plus important que dans l'ensemble de l'économie coopérative, laquelle connaît elle-même une progression des emplois supérieure à celle des entreprises privées pendant la période d'observation. Ces résultats quantitatifs et la recherche documentaire permettent d'affirmer que le tourisme coopératif a pour finalité essentielle de créer des emplois dans les territoires au Québec. D'ailleurs, certaines d'entre elles revendiquent explicitement une mission de réinsertion sociale, telle la coopérative les Mains tissées ${ }^{19}$. Le tourisme coopératif est donc territorialement orienté en matière de gouvernance, de prestations et d'échelle. Sa finalité sociale est une réponse explicite à des besoins sociaux non satisfaits. La prépondérance du statut de coopérative de solidarité implique, a priori, un fort ancrage territorial et une maitrise plus grande de la mise en tourisme. Enfin, les montants des actifs et des 
chiffres d'affaires permettent de positionner les coopératives touristiques comme de petites organisations qui dynamisent leur milieu.

La seconde dimension du tourisme coopératif, mise en exergue, concerne ses caractéristiques en amont de la production. Les outputs, c'est-à-dire les produits touristiques, apparaissent indifférenciés, a contrario des inputs, c'est-à-dire des conditions qui président à la production. La référence à l'économie sociale s'avère pertinente pour préciser ses quatre spécificités.

La première est la distribution interdite ou limitée des excédents. De ce fait, les réserves sont très conséquentes pour des coopératives ayant plusieurs années d'existence. Elles se révèlent proportionnellement plus importantes dans les coopératives touristiques, malgré leur jeunesse, que dans l'ensemble du mouvement coopératif. Elles atteignent plus de $20 \%$ des actifs. Poutant les fondateurs disposent au départ de peu de fonds initiaux, les parts sociales sont plus faibles que dans l'ensemble du mouvement coopératif. Cela signifie que ce sont principalement les résultats économiques des années d'activité qui financent le développement de l'activité coopérative. Le modèle coopératif fournit ainsi les outils favorables à la « recherche de capital et l'obtention de financements» dans le cas de "dévitalisation de certains territoires et d'absence de moyens » (Beaubien S., $2015: 56$ ).

La seconde spécificité est l'autonomie et l'indépendance de la coopérative touristique. Dans la pratique, certaines collectivités territoriales sont des membres de soutien. Toutefois, l'implication de celles-ci en tant que membres est limitée : elles ne doivent pas dépasser un tiers des administrateurs et aucune ristourne ne peut leur être attribuée. De plus, les subventions ne semblent pas suffisamment conséquentes pour contrecarrer l'autonomie et l'indépendance fixées par les statuts. Par ailleurs, les coopératives touristiques disposent d'un relatif contrôle sur la commercialisation.

La troisième spécificité concerne la gouvernance démocratique. En économie sociale, le principe démocratique, une personne $=$ une voix, s'oppose au principe ploutocratique, une action = une voix, des entreprises capitalistes. Ce principe est gravé dans le marbre par les statuts des coopératives. La recherche a confirmé que le tourisme coopératif repose sur une gouvernance démocratique majoritairement multipartite qui associe des travailleurs, des utilisateurs et des membres de soutien, ce qui implique la prise en charge par une partie de communauté de la mise en tourisme et de son propre développement.

La quatrième et dernière spécificité concerne l'hybridation des ressources. Selon Karl Polanyi (1983), les activités économiques sont encastrées (embededness) dans la sphère sociale et se déclinent selon une pluralité de modes de circulation des biens et services. Les ressources marchandes, non marchandes et non monétaires sont mobilisées dans toute économie, mais assumées, voire revendiquées, dans le cadre de l'économie sociale. Les ressources marchandes peuvent être appréhendées par la variable du chiffre d'affaires. Les ressources non marchandes, telles que les subventions, peuvent aussi être mises en évidence dans une certaine mesure. Il a été observé que celles-ci étaient généralement faibles, excepté dans le cas de la Gaspésie-Îles-de-la-Madeleine. De plus, la recherche documentaire et la revue de littérature révèlent une forte présence de bénévolat dans les coopératives touristiques, notamment pour l'aménagement de certaines structures. Retenons donc que le tourisme coopératif est une activité à dominante marchande qui repose sur une pluralité de ressources marchandes, non marchandes et non monétaires. 


\section{Discussion et conclusion. Le tourisme coopératif québécois : une forme de tourisme originale mais non « miraculeuse » pour le développement territorial}

31 L'objectif principal de recherche visait à documenter le tourisme coopératif québécois. À partir des éléments exposés, il a été possible de repérer plusieurs de ses caractéristiques :

32 1) il s'agit d'une forme de tourisme déjà ancienne, mais en forte croissance et en renouvellement institutionnel sous le statut de coopératives de solidarité ;

33 2) il est centré sur une problématique de création d'emplois, voire de réinsertion sociale ;

34 3) il présente plusieurs spécificités en amont du processus de production, notamment dans ses modalités de gouvernance et de financement.

Un autre objectif de recherche consistait à tisser des liens entre développement territorial, économie sociale et tourisme. Plusieurs auteurs (Vienney, 1994 ; Lévesque, 2007b ; Laville, 2011 ; Draperi, 2012) ont montré que l'économie sociale pouvait être mobilisée lorsque des besoins ne sont pas satisfaits par l'État ou le marché. Les protagonistes de l'économie sociale sont généralement relativement dominés socialement et s'associent pour sauvegarder des activités ou combler des besoins non satisfaits (Ibid.). Selon les résultats de la recherche, un processus de mobilisation coopératif et touristique a bien été observé dans les territoires en difficulté et ce, à différentes échelles. Ce constat ouvre des perspectives tant au niveau de la recherche que des politiques gouvernementales de développement régional ou de soutien au domaine coopératif.

L'effet de mobilisation touristique dans les territoires en difficulté pose cependant question. Les relations entre tourisme et développement territorial sont généralement traitées sous l'angle des systèmes touristiques locaux (Perret, 1992) ou de la théorie de la base revisitée, sous le qualificatif d'économie résidentielle (Davezies, 2009). La première approche induit, le plus souvent, une focalisation sur des territoires qui gagnent et sur leurs conditions de réussite. La seconde approche postule que les dynamiques de peuplement et de captation de revenus dans des territoires avec de faibles dynamiques productives seraient le fait de la consommation des populations, dont les touristes et les résidents secondaires. Si ce postulat peut expliquer le dynamisme démoéconomique de certains territoires et un possible système productivorésidentiel en particulier dans le bassin montréalais, les territoires touristiques qui gagnent des habitants, et dont les revenus sont supérieurs à la moyenne, demeurent tout de même peu nombreux au Québec. Or si la présence du tourisme coopératif est plus forte dans les territoires considérés en difficulté, cela ne semble pas suffisant pour inverser les dynamiques démoéconomiques à l'oeuvre dans ces territoires, et ce sur une période couvrant une quinzaine d'années.

L'échelle d'observation et d'analyse a également posé question : celle-ci pouvait-elle empêcher d'appréhender des dynamiques plus localisées dont les effets seraient dilués et non visibles à l'échelle régionale. En utilisant une approche originale basée sur la densité de l'offre touristique, les hauts lieux touristiques au Québec ont été repérés. Puis, nous avons observé les dynamiques de peuplement et de captation de revenus 
dans ces hauts lieux à l'aide d'une analyse cartographique. Il a été possible d'identifier une absence de corrélation entre les hauts lieux touristiques et des dynamiques démoéconomiques favorables: seulement neuf hauts lieux touristiques chevauchent des territoires en développement, selon des critères de peuplement et de captation de revenus. En conséquence de quoi, l'analyse multiscalaire nous a permis de lever les doutes sur l'éventualité d'une échelle régionale trop large qui expliquerait les résultats inattendus. L'approche multiscalaire, induite par l'approche théorique retenue, apparaît donc pertinente pour mesurer les réels effets démoéconomiques du tourisme.

Ainsi une autre considération explicative transparait: la possibilité d'un surinvestissement social du tourisme comme levier de développement. L'exemple de cette recherche et de ses postulats, notamment en ce qui concerne les dynamiques de captation de revenus et de peuplement dans les territoires mis en tourisme, met en exergue combien il est risqué d'associer trop hâtivement le tourisme et le développement. Beaucoup de travaux, dans les champs $\mathrm{du}$ tourisme et $\mathrm{du}$ développement régional, portent sur les territoires qui gagnent. Les difficultés méthodologiques et d'accès aux données expliquent possiblement la prépondérance des monographies lesquelles posent parfois des problèmes de généralisation. Si le tourisme a une capacité reconnue à transformer les lieux et à générer des dynamiques démoéconomiques, il ne constitue pas une recette qui peut être déclinée invariablement sur l'ensemble des territoires. Selon Violier (2008), le tourisme servirait, de plus en plus systématiquement, de solution de dernier recours, lorsque plus rien ne fonctionne. Il est possible que les collectivités territoriales et les instances nationales, au Québec comme ailleurs, recourent ou tentent de recourir, de manière trop systématique et peu critique au tourisme comme une avenue de développement sine qua non. Il pourrait exister un effet de mobilisation alimenté par un discours récurrent des collectivités et des organismes internationaux sur le miracle du tourisme et par le manque de recherches sur la relativisation des effets du tourisme.

En conclusion, les capacités de mobilisation et d'action collective, d'expérimentation et d'innovation sociale des coopératives de tourisme au Québec sont remarquables. Ces structures sont originales au regard des pratiques européennes de tourisme social, davantage centrées sur le statut associatif et sur l'objectif de démocratisation des vacances, et de taille plus importante (McCabe S. et al., 2012). Cependant, cette multitude de coopératives touristiques n'a pas réussi à prendre une orientation commune et ainsi créer un mouvement plus large de mobilisation impactant sur les dynamiques territoriales régionales. Bien sûr, la Fédération des Coopératives de Développement Régional du Québec (FCDRQ) et le CQCM (Conseil québécois de la coopération et de la mutualité) s'inscrivent dans un mouvement social et un effort de fédération, mais force est de constater, suite à cette recherche, que le tourisme est encore le parent pauvre du mouvement coopératif. Il apparaît peu réaliste que ces petites organisations atomisées et, plus largement le secteur touristique, laissés à euxmêmes, sans intégration à des planifications stratégiques, sans soutien étatique significatif, puissent modifier significativement le cours des trajectoires et des dynamiques des territoires en difficulté dans le sens d'un développement territorial plus viable et équitable. 


\section{BIBLIOGRAPHIE}

Beaubien, Simon, Les coopératives de solidarité en tourisme : le cas de la coopérative verte au Saguenay, Montréal, Université de Québec à Montréal, 2015.

Béguin Michelle et Pumain Denise. La représentation des données géographiques, Paris, Armand Colin, 2014, Coll. Cursus, 255 pages.

Breton, Jean-Marie (dir.), Patrimoine, tourisme, environnement et développement durable, Paris, Karthala, coll. Iles et pays d'Outre-Mer, 2010.

Caire, Gilles, « Le tourisme solidaire et équitable : « niche de solidarité » ou champ d'expérimentations d'un tourisme « socialement durable ", Économie et solidarités, n 37 (2), 2006, p. 186-202.

D’Amours, Martine, L'économie sociale au Québec. Cadre théorique, histoire, réalité et défis, Montréal : Saint-Martin, Coll. aruc. économie sociale.rqrp, 2006.

Davezies, Laurent, « L'économie locale "résidentielle" », Géographie, économie et société, 2009/1, vol. 11, 2009, Lavoisier, Pages 47-53, http://www.cairn.info/revue-geographie-economiesociete-2009-1-page-47.htm.

Delisle Marie-Andrée et Jolin Louis, Un autre tourisme est-il possible ?, Québec, Presses de l’Université du Québec, 2007, Coll. Tourisme, 2007.

Draperi, Jean-François, La république coopérative, Belgique, Larcier, 2012., Coll. Droit et économie sociale et solidaire.

Favreau, Louis, Mouvement coopératif, une mise en perspective, Québec, Presses de l'Université du Québec, 2010.

Gagnon, Christiane, « Les communautés locales face aux impacts des parcs de conservation et de l'industrie touristique ", in Gagnon Christiane (dir.) Actes du forum Tourisme viable et parcs nationaux : quel avenir pour les communautés locales ?, Sacré-Cœur-sur-le-Fjord-duSaguenaycChicoutimi, 5 et 6 juin 1998, Grir/UQAC, 1999, p. 25-40.

Gagnon, Christiane et Fortin Marie-Josée, “An assessment of social impacts of national parks on communities in Quebec, Canada”, Environmental Conservation, 26 (3), 1999, p. 200-211.

Gagnon Christiane et Gagnon Serge (dir.), L'écotourisme entre l'arbre et l'écorce. De la conservation au développement viable des territoires, Sainte-Foy, Presses de l'Université du Québec, 2007.

Iankova, Katia, « Le tourisme autochtone au Québec », Globe, Revue internationale d'études québécoises, Les modernités amérindiennes et inuite, Volume 8, Number 1, 2005, p. 85-98 Laurent, Alain (dir.), Tourisme responsable. Clé d'entrée du développement territorial durable. Guide pour la réflexion et l'action, Paris, Chronique Sociale, Coll. Comprendre la société, 2009.

Laville, Jean-Louis (dir.), L'économie solidaire, Paris, CNRS éditions, 2011, Coll. Les essentiels d'Hermès, 174 pages.

Lequin, Marie, Ecotourisme et gouvernance participative, Sainte-Foy, Presses de l'Université du Québec, Coll. Temps Libre Culture, 2001.

Lévesque, Benoît, « Un siècle et demi d'économie sociale au Québec : plusieurs configurations en présence (1850-2007) », CRISES. 2007a, CRISES//ÉNAP/ARUC-és. Pages 1 à 79. N ET0703. 
Lévesque, Benoît, « Économie plurielle et développement territorial dans la perspective du développement durable : quelques éléments théoriques de sociologie économique et de socioéconomie ", CRISES. 2007b,Coll. Etudes théoriques, N ET0705, 2007.

Lévesque Benoît et Mendell Marguerite, «L'économie sociale au Québec : éléments théoriques et empiriques pour le débat et la recherche ", Lien social et politique, $n^{\circ} 41,1999$, p. 105-118.

Lévesque Benoit et Mendell Marguerite, «L'économie sociale : diversité des définitions et des constructions théoriques, Revue Interventions Economiques, n³2, 2005, 1 juillet 2005, http:// journals.openedition.org/interventionseconomiques/852, page consultée le 05 juin 2018.

Mccabe Scott, Minnaert Lynn, Diekmann Anya, Social Tourism in Europe, Theory and Practice, Bristol, Channel View Publications, 2012.

Münkner, Hans, "Multi-stakehorder co-operatives and their legal framework", in BORZAGA Carlo et SPEAR Roger (dir.). Trends and challenges for co-operatives and social enterprises in developed and transition countries, Trento, edizioni 31, 2004, p. 49-82.

Perret, Jacques, Le développement touristique local. Les stations de sport d'hiver, Université Pierre Mendès France de Grenoble, 1992, 338 pages.

Polanyi, Karl, La Grande Transformation. Aux origines politiques et économiques de notre temps, traduit de l'anglais (États-Unis) par Maurice Angeno et Catherine Malamoud, [éd. orig. The Great Transformation, New York, Farrar \& Rinehart], Paris, Gallimard, Coll. Tel, 1983 [1944].

Collection Bibliothèque des Sciences humaines, Gallimard

Salamero, Sylvain, Le tourisme coopératif au Québec : un enjeu de développement des territoires. Thèse de doctorat en économie et en développement régional, Université de Poitiers \& Université du Québec à Chicoutimi, 2017.

Schéou, Bernard, Du tourisme durable au tourisme équitable : quelle éthique pour le tourisme de demain ?, Bruxelles, De Boeck Université, Coll. Les métiers du tourisme, 2009.

Vaillancourt, Yves, «L'économie sociale au Québec et au Canada : configurations historiques et enjeux actuels », Cahiers du Larepps, Nº8-07. ARUC ES et CRISES. 2008, p. 1-59.

Vienney, Claude, L'économie sociale, Paris, La découverte, 1994, Coll. Repères, 125 pages.

Violier, Philippe, Tourisme et développement local, Paris, Belin, 2008, Coll. Tourisme, 192 pages.

\section{NOTES}

1. Une clarification des termes communautaire et communauté s'impose dans la mesure où, au Québec, l'action communautaire recouvre un ensemble de pratiques sociales qui s'inscrit dans un large mouvement social décrit subséquemment. Le terme communauté lui renvoie, dans son acception anglo-saxonne, aux populations locales.

2. De manière générale les spécificités historiques et culturelles du Québec en Amérique du Nord sont notables: la survivance et le maintien de la langue française ainsi que le mouvement souverainiste en sont des manifestations. L'influence de la religion catholique sur l'organisation économique et sociale jusqu'aux années 1960 est suffisamment conséquente pour différencier très clairement le Québec du reste du Canada, historiquement protestant et majoritairement anglophone. Le partage des compétences fédérales\provinciales entre le gouvernement du Québec et le gouvernement fédéral, est fixé depuis l'acte constitutionnel de 1867. Les provinces ont donc des organisations économiques et sociales très différentes.

3. La première mutuelle est créée en 1789 : la Société Bienveillante du Québec. 
4. En 2015, elle comptait 500 employés, essentiellement madelinots, et réalisait 72 millions \$ (dollars canadiens) de chiffre d'affaires. Depuis 1966, la CTMA a développé progressivement des activités touristiques. Des croisières thématiques et des forfaits touristiques sont proposés, participant grandement à l'augmentation des flux touristiques dans les îles.

5. Les groupes populaires et le mouvement syndical mettent en place des centres communautaires, gérés par les populations locales qui luttent contre l'exclusion : des cliniques de santé, de cliniques juridiques, des associations coopératives d'économie familiale, des garderies, des comités de logement, des coopératives d'habitation et centres de vacances...

6. La Coopérative des travailleurs et travailleuses Premier défi, Laval a été créée en octobre 1993 avec le soutien de la Confédération des syndicats nationaux (CSN) et de la Fédération québécoise des coopératives de travail. L'objectif était de permettre aux salariés de reprendre un restaurant Saint-Hubert situé boulevard des Laurentides à Laval. Le contrat de franchise négocié s'appuye sur une redevance en fonction $d u$ volume de ventes, ce qui a permis de minimiser l'investissement des travailleurs. La coopérative, d'une soixantaine de membres sur les 90 salariés réalise depuis des excédents.

7. Une pourvoirie est une structure qui offre des services et des infrastructures en lien avec la chasse, la pêche ou la trappe. Les services consistent souvent en l'offre d'équipements, de guidages et d'hébergements.

8. Voir https://vacancesessipit.com/

9. Le Forum pour l'emploi, une initiative non gouvernementale, a impulsé une dynamique importante qui a culminé en 1989 avec un Forum «où les syndicats comptaient 486 représentants, le sociocommunautaire: 234, les coopératives: 192, les ministères: 181, les entreprises privées : 132 " (Lévesque B. et Mendell M., 1999: 15). De même Urgence Rurale, devenu ensuite Solidarité rurale lors des États généraux du monde rural en février 1991 qui ont réuni 1200 délégués, a permis la reconnaissance des groupes communautaires ruraux (Ibid) dans le développement local.

10. Voir : https://voyagescoste.ca/

11. La Loi sur les coopératives date de 1997. Les données disponibles sur les coopératives les plus récentes lors de la recherche étaient celles de 2013. Ces données sont compilées à partir des chiffres issus des rapports de gestion des coopératives non financières, transmis par le ministère de l'Économie, de la Science et de l'Innovation pour cinq séries (1997, 2001, 2006, 2011 et 2013).

12. Lorsque le quotient de localisation est supérieur à 1 , la modalité examinée de l'unité spatiale en question est supérieur à son poids dans l'ensemble des régions et est considérée comme une activité basique.

13. Le poids du tourisme dans le PIB a tendance à diminuer sur la période d'observation, soit de 1998 à 2013. Les données montrent que les volumes de nuitées, de visiteurs ou de recettes augmentent globalement au Québec, mais que l'augmentation s'infléchit dès 2002. De plus, les chiffres de l'OMT révèlent un déclin du nombre des voyageurs internationaux au Canada. Le marché intérieur prend ainsi une place de plus en plus importante par rapport au tourisme international. Les Québécois représentaient 67\% des touristes en 1998. Quinze années plus tard, le nombre de touristes québécois atteint $78 \%$ des touristes sur un total de 29 millions.

14. Le mouvement coopératif repose historiquement sur des formes unisociétaires : agriculteurs, travailleurs, commerçants ou artisans, consommateurs... Les coopératives multipartites ont été institutionnalisées en 1988 en Italie, en 1997 au Québec, en 1998 au Portugal et en 2001 en France (Münkner H., 2004). Elles ont en commun d'avoir des sociétaires hétérogènes.

15. Les régions québécoises considérées en difficulté sont les régions dont les dynamiques démographiques, productives, les taux d'emplois et de captation de revenus se situent en-dessous de la moyenne québécoise et dans une évolution défavorable pendant la période d'observation (Salaméro S., 2017). 
16. Cette concentration est observable sur l'ensemble des variables: le chiffre d'affaires, les emplois, le nombre de membres ainsi que les actifs, les parts sociales, la réserve générale, salaires et subventions.

17. La base de données 2015 de Tourisme Québec comporte les lieux d'accueil et de renseignements touristiques (240 lieux), les manifestations touristiques (800 manifestations), les attraits touristiques (4 000 attraits), les services touristiques (3 000 établissements) et les structures d'hébergement touristique (8 000 établissements).

18. La concentration des coopératives touristiques a été appréhendée à l'aide du coefficient de localisation suivant les trajectoires démoéconomiques des territoires.

19. La coopérative Les Mains tissées, propriétaire d'une auberge de jeunesse au Saguenay, a été créée en 2009 pour donner suite à une initiative individuelle. Depuis 2011, la coopérative et ses partenaires offrent l'opportunité à des jeunes sans-emploi de la région de participer à des plateaux de travail dans un objectif de réinsertion. Voir: http://www.lesmainstissees.ca/ index.php/projet-social

\section{RÉSUMÉS}

Plus de 200 coopératives touristiques œuvrent dans le secteur du tourisme et du loisir sur l'ensemble du territoire québécois. Leur succès s'inscrit dans une longue tradition d'économie sociale au Québec se développant tout au long du XX $X^{\mathrm{e}}$ siècle. Depuis la fin des années 1990, le tourisme coopératif est engagé dans un processus d'expansion et de fort renouvellement. Le nombre de coopératives touristiques a plus que doublé en vingt ans et la moitié d'entre elles ont adopté le statut de coopérative multipartite de solidarité, introduit par la Loi sur les coopératives de 1997. Ancrées territorialement, les coopératives touristiques sont centrées sur la création d'emplois locaux, la mise en commun des moyens de production et le financement de la mise en tourisme de leur territoire. Ce sont des petites entreprises en matière de chiffre d'affaires et d'actifs. Elles sont localisées principalement dans les territoires périphériques en difficulté démoéconomique, mais disposant de fortes dynamiques coopératives et touristiques préexistantes. Elles s'intègrent dans les principes de l'économie sociale, en matière de finalité sociale, de gouvernance démocratique, d'autonomie et d'hybridation des ressources. Cependant, cette multitude de coopératives touristiques n'a pas réussi à créer un mouvement plus large de mobilisation impactant sur les dynamiques territoriales régionales et promouvant une orientation commune.

Over 200 tourism cooperatives operate in the leisure industry across the Quebec territory. Their success is part of a long tradition of Quebec socio-economics which has developed throughout the 20th century. Since the end of the 90s, co-operative tourism has engaged in a process of expansion and strong renewal. The number of tourism cooperatives has more than doubled in twenty years and more than half of them have chosen the status of multi-stakeholder solidarity cooperative, introduced by the 1997 Cooperatives Law. Territorially based, the tourism cooperatives are focussed on the creation of local employment, pooling of production resources and funding for tourism development in the region. They are small businesses in terms of turnover and assets. They are mainly located in peripheral territories which have difficult socioeconomic conditions but which have strong pre-existing co-operative tourism dynamics. They fit into the principles of social economy, in terms of social purpose, democratic governance, 
autonomy and the hybridization of resources. However, this multitude of tourism cooperatives has failed to create a broader mobilisation movement impacting on regional territorial dynamics and promoting a common orientation.

Más de 200 cooperativas de turismo y ocio operan en todo el territorio quebequense. Sus éxitos forman parte de una larga tradición de desarrollo de la economía social en Quebec a lo largo del siglo XX. Desde finales de la década de 1990, el turismo cooperativo ha experimentado un proceso de expansión y de fuerte renovación. El número de cooperativas turísticas se ha más que duplicado en veinte años y la mitad de ellas han adoptado el estatuto de cooperativa de solidaridad, introducido por la Ley de Cooperativas de 1997. Las cooperativas turísticas se centran en la creación de empleos locales, la compartición de recursos productivos y el financiamiento del desarrollo del turismo en su territorio. Se trata de pequeñas empresas en términos de volumen de negocios y de activos. Se localizan principalmente en territorios periféricos con dificultades demográficas y economicas, pero con fuertes dinámicas cooperativa y turística preexistentes. Se integran en los principios de la economía social, en términos de finalidad social, de gobierno democrático, de autonomía e hibridación de los recursos. Sin embargo, esta multitud de cooperativas turísticas no ha logrado crear un movimiento de movilización más amplio que puderia influir en las dinámicas territoriales y promover una orientación común.

\section{INDEX}

Keywords : Tourism, cooperatives, Quebec, territorial development

Palabras claves: Turismo, cooperativas, Quebec, desarrollo territorial

Mots-clés : Tourisme, coopératives, Québec, développement territorial

\section{AUTEURS}

\section{SYLVAIN SALAMÉRO}

Docteur en Sciences économiques, $\mathrm{PhD}$ en Développement régional, Chargé de cours à

l'Université de Saumur-Angers, l'Université Champollion et l'Université Jean-Jaurès. sylvain@soularac.com

\section{GILLES CAIRE}

Maître de Conférences HDR (habilitation à diriger des recherches) en Sciences économiques, responsable du Master Droit et développement de l'Économie sociale et solidaire, Université de Poitiers, membre du Centre de recherche sur l'intégration économique et financière (CRIEF)Gilles.caire@univ-poitiers.fr

\section{CHRISTIANE GAGNON}

PhD en aménagement, professeure au Département des sciences humaines, UQAC (Université de Québec Chicoutimi), chercheure au Centre de recherche en développement territorial (CRDT).Christiane_Gagnon@uqac.ca 